\title{
The unidentified infrared bands and the spectrum of atomic hydrogen
}

\author{
Frédéric Zagury
}

\begin{abstract}
Fondation Louis de Broglie, 23 rue Marsoulan, 75012 Paris, France
\end{abstract}
e-mail: fzagury@wanadoo.fr

Received 14 June 2021 / Accepted 20 July 2021

\begin{abstract}
This letter compares the footprints on a spectral axis of the main unidentified infrared emission bands (UIBs) with the recombination lines of atomic hydrogen. The comparison shows striking correspondences, suggesting that atomic hydrogen may be involved in the production of UIBs.
\end{abstract}

Key words. ISM: lines and bands - ISM: general - astrochemistry

\section{Introduction}

Since the late 1960s excesses of infrared emission in the 3$12 \mu \mathrm{m}$ wavelength range have been detected in all kinds of celestial environments, including planetary and reflexion nebulae, HII regions, high latitude cirrus, and galaxies. In the 1970s, these excesses were in part resolved into five unidentified infrared bands (UIBs) centered close to 3.3, 6.3, 7.7, 8.6, and $11.2 \mu \mathrm{m}$ (Russell et al. 1978). Additional smaller emission bands have since been isolated, and it is commonly accepted that UIBs consist of multiple partially overlapping sub-components (Ricca et al. 2018, and references therein). UIBs peak in photodissociation frontier regions (Boulanger et al. 1998), in which neutral atomic hydrogen predominates. Their carrier(s) must be well mixed with interstellar gas and dust (Kahanpää et al. 2003, and references therein).

Four bands (UIBs 3.3, 6.6, 7.7, and 11.2) stand out in an overview of UIB spectra (Fig. 1 in Peeters et al. 2004). UIB 8.6 is the next most important as a red shoulder of UIB 7.7. The four main UIBs appear on top of three wave-like modulations (3.3$3.6 \mu \mathrm{m}, 5-10 \mu \mathrm{m}$, and $10-14 \mu \mathrm{m})$ of the infrared excess continuum. Neither the bands nor the underlying continuum nor their interrelations are well understood.

With only a few exceptions UIB spectra from different sources superimpose well and therefore have similar imprints on the infrared spectrum. In the following sections I identify a strong correlation between the spectral imprints of UIBs and atomic hydrogen. This previously unnoticed relationship suggests the possible involvement of atomic hydrogen in the production of UIBs.

\section{Unidentified infrared bands and the spectrum of atomic hydrogen}

The 3.3-3.6 $\mu \mathrm{m}$ infrared excess region is bounded on its red side by UIB 3.3, the narrowest of the four main UIBs. UIB 3.3 occupies the $3.2-3.35 \mu \mathrm{m}$ wavelength range (see for instance Fig. 1 of the following papers: Geballe 1997; van Diedenhoven et al. 2004; Tokunaga et al. 1991) and peaks close to $P f \delta$ (Fig. 1) at the exact position of Humphrey's series limit $H u_{\infty}(\lambda=3.282 \mu \mathrm{m})$. UIB 3.3 is generally observed in conjunction with hydrogen's Brackett and Pfund recombination lines (Geballe 1997; Mori et al. 2012; Tokunaga et al. 1991). In the observations of Mori et al., the UIB disappears in directions where no hydrogen lines are detected. UIB 3.3 is followed by a not fully resolved series of sub-bands (Nagata et al. 1988) extending up to $\sim 3.6 \mu \mathrm{m}$. The whole $3.3-3.6 \mu \mathrm{m}$ complex is thus bordered by $H u_{\infty}$ on its blue side and covers the continuum of Humphrey transitions, as represented by the gray area on the left in Fig. 1.

UIBs 6.3 and 11.2 have a similar shape that sometimes includes a small knee on its blue edge (Figs. 3a,b, Figs. 1 and 7, Figs. 3 and 4 in, respectively, Kahanpää et al. 2003; Peeters et al. 2002; van Diedenhoven et al. 2004). They run from slightly under 6-6.5 $\mu \mathrm{m}$, and from under 11-11.8 $\mu \mathrm{m}$. These features encompass the continua of hydrogen's recombination lines to $n=8$ and $n=11$, respectively (Fig. 1).

The limits of UIB 7.7 are less certain because of the difficulties of disentangling the UIB from its red UIB 8.6 shoulder and fixing the continuum. In Fig. 2 of Peeters et al. (2002), the band ranges from $\sim 7.3$ to $\sim 8.3 \mu \mathrm{m}$, as represented in Fig. 1 . Mori et al. (2012) adopted a similar decomposition (their Fig. 4). The band's spectral footprint covers hydrogen transitions to $n=$ 9. In contrast to the previous bands, UIB $8.6(8.3-8.9 \mu \mathrm{m})$ contains only a few discrete transitions.

The comparison of UIBs to hydrogen recombination lines undertaken thus far could be extended to longer wavelengths. The plateau described in Van Kerckhoven et al. (2000, Fig. 1) as occurring from shortly after $15 \mu \mathrm{m}$ to slightly after $20 \mu \mathrm{m}$, which the authors interpret "as a collection of blended emission features", exactly covers the continua of hydrogen transitions to $n=13$ and $n=14$. 


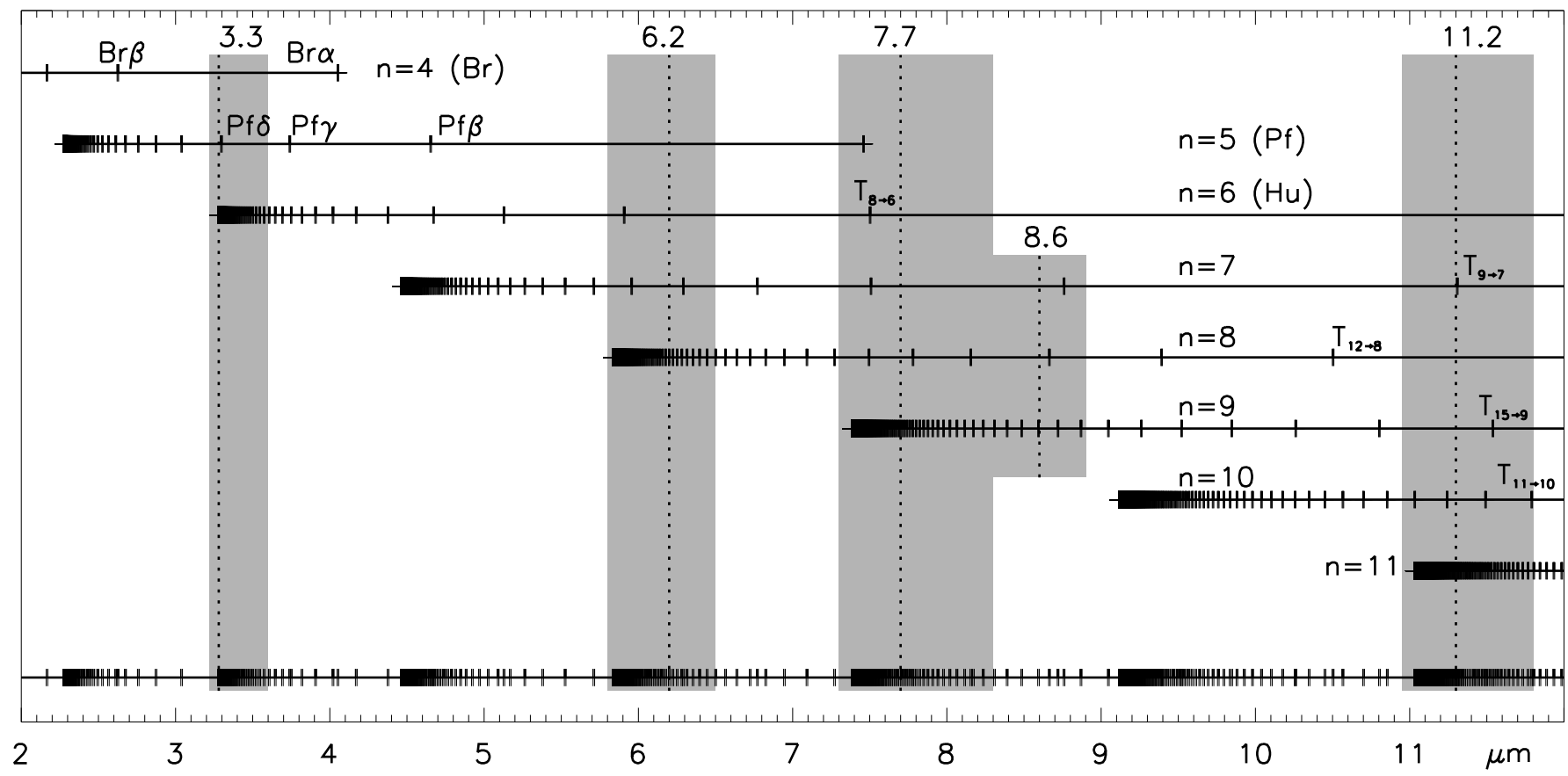

Fig. 1. UIBs and hydrogen recombination lines in the $2-12 \mu \mathrm{m}$ wavelength range. Ticks on each horizontal line segment mark transitions $T_{p \rightarrow n}$ (calculated from Rydberg's formula) from level $p>n$ to level $n$ for each $n=4,5, \ldots 11$. The bottom line gathers all hydrogen transitions between 2 and $12 \mu \mathrm{m}$. The ticks' thickness is $0.01 \mu \mathrm{m}$. Moving from red to blue on each " $n$ "-line, there is a quick convergence to a quasi-continuum of transitions from high Rydberg states, bordered on its blue side by $T_{\infty \rightarrow n}$. Shaded areas indicating the extent of UIBs are justified in the text. UIBs' conventionally agreed upon central wavelengths are represented by vertical dotted lines.

\section{Conclusion}

The near- and mid-infrared spectrum of atomic hydrogen consists in discrete transitions that tend to stack up redward of transition limits $T_{\infty \rightarrow n}(4 \leq n \leq 11)$. Merrill et al. (1975) and Geballe et al. (1985) independently noted a good match between the 3.3-3.6 $\mu \mathrm{m}$ infrared excess region and hydrogen's Humphrey $(n=5)$ transitions, but both groups of scholars dismissed the existence of any correlation ${ }^{1}$. However, Fig. 1 shows a close relationship extending well beyond a single coincidence between hydrogen's recombination spectrum from high Rydberg states and the spectrum of UIBs.

Up to the present the positions of UIBs on the spectrum have mainly been assigned to $\mathrm{C}-\mathrm{C}$ and $\mathrm{C}-\mathrm{H}$ stretches and $\mathrm{C}-\mathrm{H}$ in and out of plane bends produced by polycyclic aromatic hydrocarbon (PAH) molecules (Peeters et al. 2002, 2004). But to date the lack of an exact match between PAH transitions and UIB positions on the spectrum has hampered the firm identification of any UIB carrier. The striking overlap of UIB imprints with the spectrum of hydrogen could, in this regard, impose new constraints on the search for the carrier(s) of UIBs. Among other carrier(s) that have been suggested in the literature, Rydberg matter (atoms or molecules, including hydrogen atoms, excited to high Rydberg states) proposed by Holmlid (2000) provides the closest match to the present finding.

\section{References}

Boulanger, F., Boissel, P., Cesarsky, D., \& Ryter, C. 1998, A\&A, 339, 194 Geballe, T. R. 1997, ASPCS, 122, 1997

Geballe, T. R., Lacy, J. H., Persson, S. E., et al. 1985, ApJ, 292, 500 Holmlid, L. 2000, A\&A, 358, 276

Kahanpää, J., Mattila, K., Lehtinen, K., et al. 2003, A\&A, 405, 999 Merrill, K. M., Soifer, B. T., \& Russell, R. W. 1975, ApJ, 200, L37

Mori, T. I., Sakon, I., Onaka, T., et al. 2012, ApJ, 744, 68

Nagata, T., Tokunaga, A. T., Sellgren, K., et al. 1988, ApJ, 326, 157

Peeters, E., Hony, S., van Kerckhoven, C., et al. 2002, A\&A, 390, 1089

Peeters, E., Allamandola, L. J., Hudgins, D. M., et al. 2004, ASPCS, 309, 141

Ricca, A., Bauschlicher, C. W., Jr, Roser, J. E., \& Peeters, E. 2018, ApJ, 854, 115

Russell, R. W., Soifer, B. T., \& Willner, S. P. 1978, ApJ, 220, 568

Tokunaga, A. T., Sellgreen, K., Smith, R. G., et al. 1991, ApJ, 380, 452

van Diedenhoven, B., Peeters, E., Van Kerckhoven, C., et al. 2004, ApJ, 611, 928

Van Kerckhoven, C., Hony, S., Peeters, E., et al. 2000, A\&A, 357, 1013

\footnotetext{
1 Merrill et al. (1975) for instance argued that hydrogen transition $H u_{\infty}$ would be "significantly larger than predicted by recombination theory".
} 